\title{
GRB 011121: A MASSIVE STAR PROGENITOR
}

\author{
P. A. Price,${ }^{1,2}$ E. Berger,${ }^{2}$ D. E. Reichart,${ }^{2}$ S. R. Kulkarni, ${ }^{2}$ S. A. Yost,${ }^{2}$ R. Subrahmanyan, ${ }^{3}$ R. M. Wark, ${ }^{3}$ \\ M. H. Wieringa, ${ }^{3}$ D. A. Frail, ${ }^{2,4}$ J. Bailey, ${ }^{5}$ B. Boyle, ${ }^{5}$ E. Corbett,${ }^{5}$ K. Gunn, ${ }^{6}$ S. D. Ryder, ${ }^{5}$ \\ N. Seymour, ${ }^{6}$ K. Koviak, ${ }^{7}$ P. McCarthy, ${ }^{7}$ M. Phillips, ${ }^{7}$ T. S. Axelrod, ${ }^{1}$ J. S. Bloom, ${ }^{2}$ \\ S. G. Djorgovski, ${ }^{2}$ D. W. Fox ${ }^{2}$ T. J. Galama, ${ }^{2}$ F. A. Harrison, ${ }^{2}$ K. Hurley, ${ }^{8}$ \\ R. Sari, ${ }^{9}$ B. P. Schmidt, ${ }^{1}$ M. J. I. Brown, ${ }^{10}$ T. Cline, ${ }^{11}$ F. Frontera, ${ }^{12}$ \\ C. Guidorzi, ${ }^{13}$ AND E. Montanari ${ }^{13}$ \\ Received 2002 March 26; accepted 2002 May 2; published 2002 May 20
}

\begin{abstract}
Of the cosmological gamma-ray bursts, GRB 011121 has the lowest redshift, $z=0.36$. More importantly, the multicolor excess in the afterglow detected in the Hubble Space Telescope (HST) light curves is compelling observational evidence of an underlying supernova. Here we present near-infrared and radio observations of the afterglow, and from our comprehensive afterglow modeling, we find evidence favoring a wind-fed circumburst medium. Lacking X-ray data, we are unable to conclusively measure the mass-loss rate, $M$, but obtain an estimate, $\dot{M} \sim 2 \times 10^{-7} / v_{w 3} M_{\odot} \mathrm{yr}^{-1}$, where $v_{w 3}$ is the speed of the wind from the progenitor in units of $10^{3} \mathrm{~km} \mathrm{~s}^{-1}$. This $\dot{M}$ is similar to that inferred for the progenitor of the Type Ibc supernova SN 1998bw that has been associated with the peculiar burst GRB 980425. Our data, taken in conjunction with the HST results of Bloom et al., provide a consistent picture: the long-duration GRB 011121 had a massive star progenitor that exploded as a supernova at about the same time as the gamma-ray burst event. Finally, we note that the gamma-ray profile of GRB 011121 is similar to that of GRB 980425.
\end{abstract}

Subject heading: gamma rays: bursts

\section{INTRODUCTION}

On 2001 November 21 at 18:47:21 UT, GRB 011121 was detected and localized by the Italian-Dutch satellite BeppoSAX (Piro 2001). The localization was further improved by the Interplanetary Network (IPN; Hurley et al. 2001), and an optical transient was identified by Wyrzykowski, Stanek, \& Garnavich (2001). Spectroscopy of the transient revealed emission lines interpreted as arising from the host galaxy at a redshift of $z=0.36$ (Infante et al. 2001).

Low-redshift gamma-ray bursts (GRBs) are particularly valuable in uncovering the origin of GRBs. If GRBs result from the death of massive stars, then it is reasonable to expect an underlying supernova (SN). Bloom et al. (1999) attributed a late-time red excess seen in the afterglow emission of GRB

\footnotetext{
${ }^{1}$ Research School of Astronomy and Astrophysics, Mount Stromlo Observatory, Cotter Road, Weston Creek, Canberra, ACT 2611, Australia.

${ }^{2}$ Palomar Observatory, MS 105-24, California Institute of Technology, Pasadena, CA 91125.

${ }^{3}$ Australia Telescope National Facility, CSIRO, P.O. Box 76, Epping, NSW 1710, Australia.

${ }^{4}$ National Radio Astronomy Observatory, P.O. Box O, 1003 Lopezville Road, Socorro, NM 87801.

${ }^{5}$ Anglo-Australian Observatory, P.O. Box 296, Epping, NSW 1710, Australia.

${ }^{6}$ Department of Physics and Astronomy, University of Southampton, Highfield, Southampton SO17 1BJ, UK.

${ }^{7}$ Carnegie Observatories, 813 Santa Barbara Street, Pasadena, CA 91101.

${ }^{8}$ University of California at Berkeley, Space Sciences Laboratory, Grizzly Peak at Centennial Drive, Berkeley, CA 94720-7450.

${ }^{9}$ Theoretical Astrophysics, MS 130-33, California Institute of Technology, Pasadena CA, 91125.

${ }^{10}$ National Optical Astronomy Observatory, P.O. Box 26732, 950 North Cherry Avenue, Tucson, AZ 85726-6732.

${ }^{11}$ NASA Goddard Space Flight Center, Code 661, Greenbelt, MD 20771.

${ }^{12}$ Istituto Tecnologie e Studio Radiazioni Extraterrestri, CNR, Via Gobetti, 101, 40129 Bologna, Italy.

${ }^{13}$ Dipartimento di Fisica, Universita di Ferrara, Via Paradiso, 12, 44100 Ferrara, Italy.
}

980326 to an underlying SN. This result triggered searches for similar excesses with no clear success save GRB 970228 (Reichart 1999; Galama et al. 2000). The low redshift is critical to such searches since the SN contribution is expected to exhibit strong absorption below $4000 \AA$ in the rest frame (see Bloom et al. 1999).

Given this motivation, we triggered a sequence of multicolor and multiepoch Wide Field Planetary Camera 2 observations with the Hubble Space Telescope (HST). Garnavich et al. (2002) noted that the $R$-band flux of the first epoch of the $H S T$ observations was significantly in excess of the extrapolation of the power-law decay of the early ground-based optical afterglow and attributed this to an underlying SN component. In Bloom et al. (2002, hereafter Paper I), we presented four-epoch multicolor HST light curves and show that the data are readily explained by an underlying SN similar to SN 1998bw (Galama et al. 1998), except fainter by about $\frac{2}{3}$ mag.

This GRB-SN link is an essential expectation in the collapsar model (Woosley 1993), in which GRBs result from the death of certain massive stars. An essential consequence of any massive star origin for GRBs, as noted by Chevalier \& Li (1999), is a circumburst medium fed by the inevitable and copious mass loss suffered by massive stars throughout their lives. Afterglow observations are well suited to determining not only the geometry of the explosion but also the distribution of circumburst matter. Despite several attempts (e.g., Frail et al. 2000; Jaunsen et al. 2001; Berger et al. 2001) to identify afterglows evolving in a wind-fed medium (density, $\rho \propto r^{-s}$ with $s \sim 2$; here $r$ is the distance from the explosion site), unambiguous evidence of this signature of a massive star progenitor has yet to be revealed.

Here we present the gamma-ray profile and near-infrared (NIR) and radio observations of the afterglow of GRB 011121. We model the afterglow data and find a good case for a windfed circumburst medium. Thus, the totality of the data-the $H S T$ optical light curves and multiwavelength (radio, NIR, and 


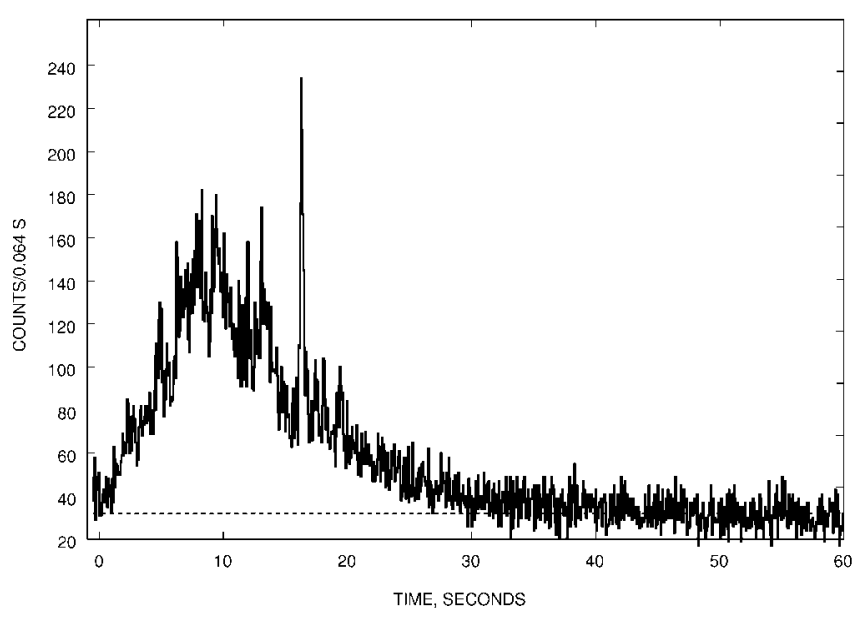

FIG. 1.-Time history of GRB 011121 in the $25-150 \mathrm{keV}$ energy range, as observed by Konus-Wind. The dotted line gives the background rate. Zero on the time axis corresponds to an Earth crossing time of $67630.899 \mathrm{~s}$.

optical) data-now support a massive star progenitor for this GRB.

\section{OBSERVATIONS}

Gamma rays.-GRB 011121 was observed by numerous spacecraft in the IPN: Ulysses, BeppoSAX (Gamma-Ray Burst Monitor), High Energy Transient Explorer 2 (French Gamma Telescope), Mars Odyssey (High-Energy Neutron Detector) and Konus-Wind. The light curve, as observed by Konus-Wind, is shown in Figure 1. The $T_{90}$ duration, as determined from the Ulysses data, was $28 \mathrm{~s}$, placing this event in the class of "long bursts." The peak flux in the $25-100 \mathrm{keV}$ range, over $0.25 \mathrm{~s}$, was $2.4 \times 10^{-6} \mathrm{ergs} \mathrm{cm}^{-2} \mathrm{~s}^{-1}$, and the fluence was $2.4 \times$ $10^{-5}$ ergs $\mathrm{cm}^{-2}$.

NIR.-We observed the afterglow in the NIR with the newly commissioned Infrared Imager and Spectrograph 2 (IRIS2) on the Anglo-Australian Telescope (AAT), the Wide Field Infrared Camera on the du Pont $2.5 \mathrm{~m}$ telescope, and the IRCam on the Walter Baade $6.5 \mathrm{~m}$ telescope in $J$ and $K_{s}$ filters. The images were dark-subtracted, flat-fielded, sky-subtracted, and combined using DIMSUM ${ }^{14}$ in IRAF. Point-spread function fitting photometry of the afterglow using DAOPHOT (Stetson 1987) was performed relative to point sources in the field. Our multiple calibrations are consistent with each other, and we estimate the systematic error to be less than $0.05 \mathrm{mag}$ (see Table 1).

Radio.-We initiated observations of GRB 011121 with the Australia Telescope Compact Array (ATCA) starting on 2001 November 22.58 UT (see Table 2). The data were reduced and imaged using the MIRIAD software package.

\section{MODELING THE AFTERGLOW}

\subsection{Dust Extinction}

In Figure 2, we display the optical/NIR spectrum of GRB 011121. The apparent curvature in the spectrum indicates a large magnitude of dust extinction. In view of this, estimating the dust extinction accurately is critical not only for the afterglow modeling but also for the SN modeling of the HST light curves (Paper I).

From the IR dust maps (Schlegel, Finkbeiner, \& Davis 1998),

\footnotetext{
${ }^{14}$ See http://iraf.noao.edu/iraf/ftp/contrib/dimsumV2/dimsum.readme.
}

TABLE 1

NIR ObSERVAtions of the Afterglow of GRB 011121

\begin{tabular}{|c|c|c|c|}
\hline $\begin{array}{c}\text { Date } \\
\text { (2001 UT) }\end{array}$ & Filter & Magnitude & Telescope \\
\hline Nov 22.3560 & $J$ & $17.852 \pm 0.045$ & $\mathrm{dP}$ \\
\hline Nov 22.3573 & $J$ & $17.730 \pm 0.037$ & $\mathrm{dP}$ \\
\hline Nov 22.3587 & $J$ & $17.763 \pm 0.044$ & $\mathrm{dP}$ \\
\hline Nov 22.3600 & $J$ & $17.801 \pm 0.040$ & $\mathrm{dP}$ \\
\hline Nov 22.3614 & $J$ & $17.821 \pm 0.037$ & $\mathrm{dP}$ \\
\hline Nov 22.3627 & $J$ & $17.799 \pm 0.039$ & $\mathrm{dP}$ \\
\hline Nov 22.3641 . & $J$ & $17.785 \pm 0.035$ & $\mathrm{dP}$ \\
\hline Nov $22.3654 \ldots \ldots$ & $J$ & $17.770 \pm 0.036$ & $\mathrm{dP}$ \\
\hline Nov 22.3667 & $J$ & $17.795 \pm 0.041$ & $\mathrm{dP}$ \\
\hline Nov 22.3681 & $J$ & $17.739 \pm 0.038$ & $\mathrm{dP}$ \\
\hline Nov 22.7177 & $J$ & $18.352 \pm 0.100$ & AAT \\
\hline Nov 23.3193 & $J$ & $19.463 \pm 0.068$ & $\mathrm{dP}$ \\
\hline Nov 28.5 & $J$ & $21.291 \pm 0.282$ & Baade \\
\hline Nov 22.3178 & $K$ & $15.959 \pm 0.045$ & $\mathrm{dP}$ \\
\hline Nov 22.3194 & $K$ & $15.987 \pm 0.040$ & $\mathrm{dP}$ \\
\hline Nov 22.3211 . & $K$ & $15.908 \pm 0.037$ & $\mathrm{dP}$ \\
\hline Nov 22.3227 & $K$ & $15.994 \pm 0.040$ & $\mathrm{dP}$ \\
\hline Nov 22.3244 & $K$ & $15.958 \pm 0.040$ & $\mathrm{dP}$ \\
\hline Nov 22.3263 & $K$ & $16.002 \pm 0.041$ & $\mathrm{dP}$ \\
\hline Nov 22.3279 & $K$ & $16.006 \pm 0.041$ & $\mathrm{dP}$ \\
\hline Nov 22.3296 & $K$ & $16.003 \pm 0.039$ & $\mathrm{dP}$ \\
\hline Nov 22.3296 & $K$ & $16.003 \pm 0.039$ & $\mathrm{dP}$ \\
\hline Nov 22.3313 & $K$ & $15.981 \pm 0.037$ & $\mathrm{dP}$ \\
\hline Nov $22.3329 \ldots \ldots$ & $K$ & $16.053 \pm 0.039$ & $\mathrm{dP}$ \\
\hline Nov 22.3349 & $K$ & $16.039 \pm 0.040$ & $\mathrm{dP}$ \\
\hline Nov $22.3365 \ldots \ldots$ & $K$ & $15.997 \pm 0.039$ & $\mathrm{dP}$ \\
\hline Nov $22.3382 \ldots \ldots$ & $K$ & $16.120 \pm 0.041$ & $\mathrm{dP}$ \\
\hline Nov $22.3398 \ldots \ldots$ & $K$ & $15.996 \pm 0.063$ & $\mathrm{dP}$ \\
\hline Nov $22.3454 \ldots \ldots$ & $K$ & $16.027 \pm 0.038$ & $\mathrm{dP}$ \\
\hline Nov 22.3470 & $K$ & $16.069 \pm 0.036$ & $\mathrm{dP}$ \\
\hline Nov 22.3487 & $K$ & $16.100 \pm 0.042$ & $\mathrm{dP}$ \\
\hline Nov 22.3503 & $K$ & $16.015 \pm 0.043$ & $\mathrm{dP}$ \\
\hline Nov 22.3520 . & $K$ & $16.098 \pm 0.043$ & $\mathrm{dP}$ \\
\hline Nov $22.4771 \ldots \ldots$ & $K$ & $16.421 \pm 0.041$ & AAT \\
\hline Nov $22.4954 \ldots .$. & $K$ & $16.537 \pm 0.041$ & AAT \\
\hline Nov $22.5126 \ldots \ldots$ & $K$ & $16.495 \pm 0.035$ & AAT \\
\hline Nov $22.6066 \ldots . .$. & $K$ & $16.605 \pm 0.058$ & AAT \\
\hline Nov $22.6169 \ldots \ldots$ & $K$ & $16.788 \pm 0.042$ & AAT \\
\hline Nov 22.6397 & $K$ & $16.782 \pm 0.038$ & AAT \\
\hline Nov $22.6506 \ldots \ldots$ & $K$ & $16.862 \pm 0.036$ & AAT \\
\hline Nov 22.6612 & $K$ & $17.019 \pm 0.052$ & AAT \\
\hline Nov $22.6716 \ldots .$. & $K$ & $16.852 \pm 0.039$ & AAT \\
\hline Nov $22.6822 \ldots \ldots$ & $K$ & $17.035 \pm 0.083$ & AAT \\
\hline Nov $22.7272 \ldots \ldots$ & $K$ & $17.005 \pm 0.051$ & AAT \\
\hline Nov $22.7384 \ldots \ldots$ & $K$ & $17.087 \pm 0.079$ & AAT \\
\hline Nov $23.3336 \ldots \ldots$ & $K$ & $17.924 \pm 0.051$ & $\mathrm{dP}$ \\
\hline Nov $28.7092 \ldots .$. & $K$ & $19.346 \pm 0.234$ & AAT \\
\hline
\end{tabular}

Note.-Observations at the du Pont $(\mathrm{dP}) 2.5 \mathrm{~m}$ were made by K. Koviak; observations at the AAT were made by S. D. Ryder (Nov 22) and K. Gunn (Nov 28); and observations at the Baade telescope were made by M. Phillips. The following reference stars were used. For $K_{s}$ observations on the AAT, we observed UKIRT Faint Standards FS 7, 11, and 13 (Hawarden et al. 2001) on 2001 November 28. SJ 9113 (Persson et al. 1998) was observed at the du Pont telescope on 2001 November 23. We assumed an atmospheric extinction coefficient of 0.09 mag per air mass in $K$ for the du Pont observations and that the color terms were negligible. We used the reference stars calibrated by Phillips et al. (2001) to calibrate our $J$-band observations. The AAT measurement of November 28 is contaminated by both the host and the nearby star.

we estimate the visual extinction $A_{V} \approx 1.6 \mathrm{mag}$. However, it appears that the line of sight to the afterglow passes through the edge of a dust cloud $\sim 45^{\prime}$ in extent. Fortunately, the availability of both the optical and NIR afterglow data allow us to directly estimate the extinction along this line of sight.

We make the reasonable assumption that the late-time ( $>1$ day) optical/NIR afterglow follows a standard power-law 
TABLE 2

Radio ObSERVATIONS OF GRB 011121 Made WITH ATCA ${ }^{\mathrm{a}}$

\begin{tabular}{ccc}
\hline \hline $\begin{array}{c}\text { Epoch }^{\mathrm{b}} \\
(\mathrm{UT})\end{array}$ & $\begin{array}{c}\nu_{0}^{\mathrm{c}} \\
(\mathrm{GHz})\end{array}$ & $\begin{array}{c}S \pm \sigma^{\mathrm{d}} \\
(\mu \mathrm{Jy})\end{array}$ \\
\hline 2001 Nov $25.20 \ldots \ldots$ & 4.80 & $240 \pm 70$ \\
2001 Nov $28.64 \ldots \ldots$. & 4.80 & $510 \pm 38$ \\
2001 Dec $6.80 \ldots \ldots$. & 4.80 & $350 \pm 42$ \\
2001 Dec $15.80 \ldots \ldots$. & 4.80 & $250 \pm 34$ \\
2001 Dec $22.90 \ldots \ldots$. & 4.80 & $-99 \pm 49$ \\
2002 Jan $23.85 \ldots \ldots$. & 4.80 & $320 \pm 38$ \\
2001 Nov $22.83 \ldots \ldots$. & 8.70 & $210 \pm 40$ \\
2001 Nov $25.08 \ldots \ldots$. & 8.70 & $450 \pm 130$ \\
2001 Nov $28.64 \ldots \ldots$. & 8.70 & $610 \pm 39$ \\
2001 Dec $6.80 \ldots \ldots$. & 8.70 & $220 \pm 58$ \\
2001 Dec $15.80 \ldots \ldots$. & 8.70 & $274 \pm 37$ \\
2001 Dec 22.90 $\ldots \ldots$. & 8.70 & $237 \pm 46$ \\
2002 Jan 23.85 $\ldots \ldots .$. & 8.70 & $-99 \pm 47$ \\
\hline
\end{tabular}

${ }^{a}$ All observations were obtained using the continuum mode and a $128 \mathrm{MHz}$ bandwidth. The flux calibration was performed using PKS B1934-638, while the phase was monitored using PKS B1057797.

${ }^{\mathrm{b}}$ UT date of the start of each observation.

${ }^{c}$ Center frequency.

${ }^{\mathrm{d}}$ Peak flux density at the best-fit position of the radio transient, with the error given as the rms noise on the image.

model, $F_{\nu} \propto t^{-\alpha} \nu^{-\beta}$. We apply the parametric extinction curves of Cardelli, Clayton, \& Mathis (1989) and Fitzpatrick \& Massa (1988) along with the interpolation calculated by Reichart (2001). Thanks to the abundance of our NIR data, which suffer little extinction, we can break the degeneracy between $\beta$ and $A_{V}$.

In addition to our own measurements, we have included those reported in the literature (and noted in Fig. 2). Since latetime measurements are increasingly dominated by an uncertain mix of the afterglow, the host galaxy, and the nearby star B (Paper I), we restrict the analysis to data obtained over the first 2 days.

Our best fit has an unacceptable $\chi^{2}=66$ for 48 degrees of freedom, but this is mainly dominated by outliers, particularly data taken in poor seeing where the afterglow is blended with star B. Inserting an additional 3\% error to each of our measurements decreases the $\chi^{2}$ to match the number of degrees of freedom. The additional error term, while ad hoc, is reasonable given the variety of telescopes and reduction techniques in our data set.

We find $\alpha=1.66 \pm 0.06, \beta=0.76 \pm 0.15$, and $A_{V}=$ $1.16 \pm 0.25 \mathrm{mag}$. The latter is distinctly lower than that deduced from the dust maps (see above). With our data, we are unable to constrain the type of extinction curve (e.g., Milky Way, LMC, SMC, etc.). We note that we have not considered extinction within the host galaxy.

\subsection{Afterglow Models}

Armed with $\alpha$ and $\beta$, we now consider three afterglow models: (1) isotropic expansion into a homogeneous medium (Sari, Piran, \& Narayan 1998), (2) isotropic expansion into a windstratified medium (Chevalier \& Li 1999), and (3) collimated expansion into a homogeneous or wind-stratified medium (Sari, Piran, \& Halpern 1999). The models can be distinguished by a closure relation, $\alpha+b \beta+c=0$. These closure relations are due to the dependence of both $\alpha$ and $\beta$ on the electron energy distribution index, $p$, and the values of $b$ and $c$ depend on the

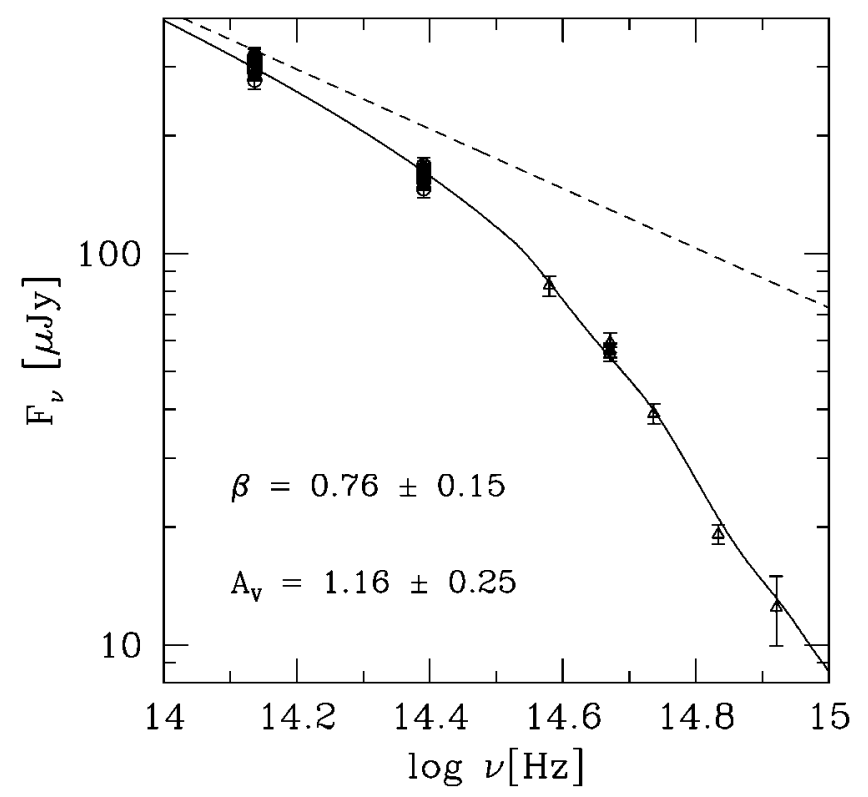

FIG. 2.-Optical and NIR spectral flux distribution of the afterglow of GRB 011121 at 0.5 days, based on measurements presented here (circles) and from the literature (triangles; Olsen et al. 2001; Stanek \& Wyrzykowski 2001). Measurements taken within $0.5 \pm 0.1$ days have been transformed to 0.5 days using the best-fit model. The solid line indicates our best fit to the data, using a power-law model plus foreground extinction. The dashed line is the intrinsic spectrum of the afterglow.

location of the cooling frequency, $\nu_{c}$, relative to the optical/ NIR frequency, $\nu_{O}$, at the epoch of the observations.

As can be seen from Table 3, models with isotropic expansion into an homogeneous medium (or, equivalently, a jet that becomes apparent on a timescale longer than the epochs of the optical/NIR data used here, $t_{j} \gtrsim 2$ days) are ruled out by the closure relations at more than $2 \sigma$ significance. Two models produce closure consistent with zero: (1) a wind model with $\nu_{c}>\nu_{O}$ (effective epoch day 1) and $p=2.55 \pm 0.08$ and (2) a fully developed jet at the time of the first optical observation, $t_{j}<0.5$ days, with $\nu_{c}<\nu_{O}$ and $p=1.66 \pm 0.06$.

The radio measurements, however, do not show any sign of a decay until at least $\sim 7$ days after the burst (Fig. 3). The rising centimeter-band flux prior to this time indicates that the jet break is not at early times, and hence the first model, i.e., the

TABLE 3

Afterglow Model Testing

\begin{tabular}{cccrc}
\hline \hline Model & $\nu_{c}$ & $(b, c)$ & \multicolumn{1}{c}{ Closure } & $p$ \\
\hline ISM $\ldots \ldots .$. & $\mathrm{B}$ & $(-3 / 2,0)$ & $1.04 \pm 0.47$ & $3.21 \pm 0.08$ \\
& $\mathrm{R}$ & $(-3 / 2,1 / 2)$ & $2.04 \pm 0.47$ & $2.88 \pm 0.08$ \\
Wind $\ldots . .$. & $\mathrm{B}$ & $(-3 / 2,-1 / 2)$ & $0.04 \pm 0.47$ & $2.55 \pm 0.08$ \\
& $\mathrm{R}$ & $(-3 / 2,1 / 2)$ & $2.04 \pm 0.47$ & $2.88 \pm 0.08$ \\
Jet $\ldots \ldots \ldots$. & $\mathrm{B}$ & $(-2,-1)$ & $-0.86 \pm 0.31$ & $1.66 \pm 0.06$ \\
& $\mathrm{R}$ & $(-2,0)$ & $0.14 \pm 0.31$ & $1.66 \pm 0.06$ \\
\hline
\end{tabular}

NoTE.-Calculation of the closure relations $\alpha+b \beta+c$ for a variety of afterglow models. A successful model will have a value of zero for the closure relation. The interstellar medium (ISM) and wind models are for isotropic expansion in an homogeneous and wind-stratified medium, respectively. The jet model is for collimated expansion, with the jet break time before the first observations were made. The relations are dependent on the assumed location of the cooling frequency, $\nu_{c}$, relative to the optical and NIR bands, $\nu_{O}$ : the case $\nu_{c}>\nu_{O}$ is denoted by "B" (for blueward) and $\nu_{c}<\nu_{O}$ by "R" (for redward); $p$ is the electron energy power-law index. 


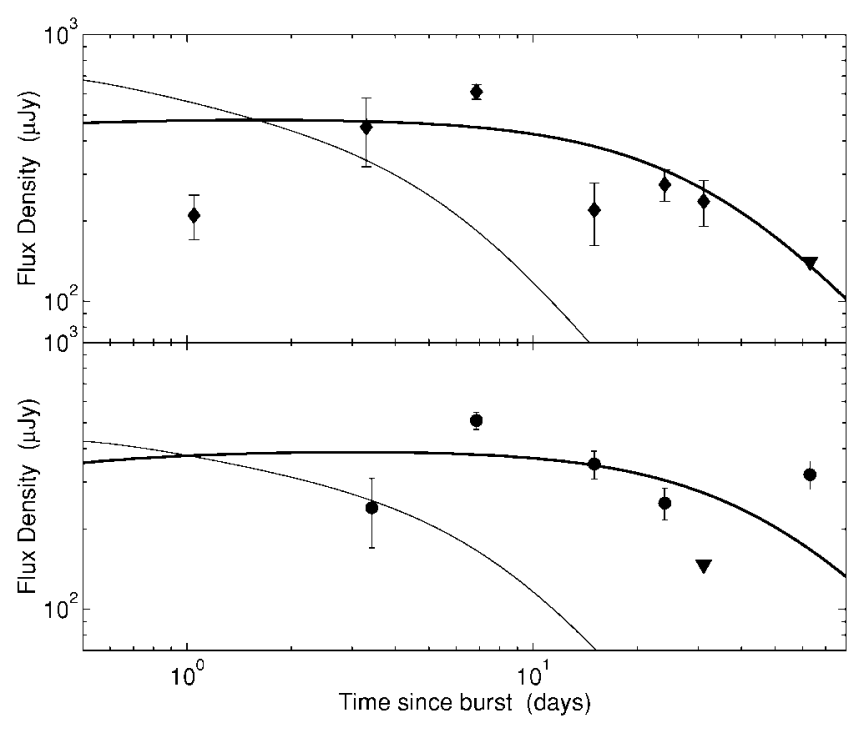

FIG. 3.-Radio light curve of the afterglow of GRB 011121. The upper panel is $8.7 \mathrm{GHz}$; the lower panel is $4.8 \mathrm{GHz}$. The thick solid line is our wind model, the thin solid line is a representative jet model, which is clearly excluded by the data. The radio data exhibit strong modulation due to interstellar scintillation and, as a result, deviate from our model by more than $1 \sigma$. This will be addressed in a future paper.

jet model, is ruled out. This then leaves us with the second model, i.e., the wind model.

\subsection{A Wind Model}

The multiwavelength data, radio through optical, are analyzed by considering the evolution of the broadband synchrotron spectrum. In addition to $\nu_{c}, p$, and $A_{V}$, we need to consider the selfabsorption frequency, $\nu_{a}$, and the peak frequency, $\nu_{m}$, as well as the peak flux, $F_{\nu, m}$. These parameters are estimated from the data and can be inverted to yield physical quantities, i.e., the energy of the fireball, the density of the ambient medium, and the fractions of energy in the electrons, $\epsilon_{e}$, and magnetic field, $\epsilon_{B}$. An example of this approach can be found in Berger et al. (2001). The density is parameterized by $A_{*}$, which is defined through $A=\dot{M} / 4 \pi v_{w}=5 \times 10^{11} A_{*} \mathrm{~g} \mathrm{~cm}^{-1}$. (see Chevalier \& Li 1999), where $v_{w}$ is the wind speed and $\dot{M}$ is the mass-loss rate. The normalization of $A_{*}=1$ applies for a typical Wolf-Rayet wind speed of $10^{3} \mathrm{~km} \mathrm{~s}^{-1}$ and $M=10^{-5} M_{\odot} \mathrm{yr}^{-1}$.

Given the sparse data, we prefer to undertake the model fitting in an evolutionary approach rather than performing a blind $\chi^{2}$-minimization search. For example, we fix the values of $p$ and $A_{V}$ to that determined earlier since the radio data have little bearing on these parameters. Next, we know that $\nu_{c}>\nu_{O}$, but there are no available data in the X-ray band to actually constrain the value of $\nu_{c}$. We therefore use $\nu_{c} \approx 10^{15} \mathrm{~Hz}$ since this is effectively the lowest value the cooling frequency can have in this model. We will, at a later point, revisit this issue and examine the consequences of increasing $\nu_{c}$.

The remaining free parameters, ${ }^{15} \nu_{a}, \nu_{m}$, and $F_{\nu, m}$, are relatively easy to constrain for the following reasons. The value of $F_{\nu, m}$ determines the overall scaling in both the optical/NIR and radio bands and is therefore constrained by these two sets of data. The value of $\nu_{m}$ is constrained by the turnover in the radio light curves (since $\nu_{m}$ passes through the radio bands at $t \approx 7$ days;

\footnotetext{
${ }^{15}$ Unless otherwise stated, all time-dependent parameters are evaluated at epoch 1 day, e.g., $\nu_{m} \equiv \nu_{m}(t=1$ day $)$.
}

see Fig. 3) as well as by the flux density of the optical/NIR light curves (since for a given value of $F_{\nu, m}$, the flux density in the optical/NIR band is determined by $\nu_{m}$ ).

Finally, $\nu_{a}$ is constrained by the spectral slope between the two centimeter bands. The comparable flux between 4.8 and 8.7 GHz suggests that $\nu_{a}<4.8 \mathrm{GHz}$. An independent constraint on $\nu_{a}$ is also provided by the following equation from Sari \& Esin (2001):

$$
\begin{aligned}
C= & 0.06(1+z)^{4} t_{\mathrm{day}}^{4} d_{L, 28}^{-2} \eta\left(\frac{\nu_{a}}{\mathrm{GHz}}\right)^{10 / 3}\left(\frac{\nu_{m}}{10^{13} \mathrm{~Hz}}\right)^{13 / 6} \\
& \times\left(\frac{\nu_{c}}{10^{14} \mathrm{~Hz}}\right)^{3 / 2}\left(\frac{F_{\nu, m}}{\mathrm{mJy}}\right)^{-1} \leq 0.25
\end{aligned}
$$

where $\eta=\min \left[\left(\nu_{c} / \nu_{m}\right)^{-(p-2) / 2}, 1\right]$ is the fraction of the electron energy radiated away.

We find that $F_{\nu, m} \approx 3 \mathrm{mJy}, \nu_{c} \approx 10^{15} \mathrm{~Hz}, \nu_{m} \approx 2 \times 10^{12} \mathrm{~Hz}$, and $\nu_{a} \approx 1.4 \mathrm{GHz}$ provide an adequate description of the afterglow data. From these parameters, we obtain $A_{*} \sim 0.02$ and find that inverse Compton (IC) cooling is marginally important.

Despite the fact that $\nu_{c}$ and $\nu_{a}$ are not observationally constrained, there are two considerations that lead us to believe that the above model is not far from the correct one: (1) as seen from equation (1), $\nu_{a}$ cannot be higher than $1.9 \mathrm{GHz}$ since otherwise $\nu_{c}$ will fall below $10^{15} \mathrm{~Hz}$, in contrast to the observations, and (2) in all cases with sufficient radio data to observationally constrain $\nu_{a}$, we obtain $\nu_{a} \sim$ a few GHz.

If we neglect the latter argument, then the value of $A_{*}$ is not well determined. Nevertheless, we can place limits based on our data. The limits depend on the importance of IC cooling: under the low IC solution, $A_{*} \approx 0.006\left(\nu_{a} / 1.4 \mathrm{GHz}\right)^{-5 / 3} \times$ $\left(\nu_{c} / 10^{15} \mathrm{~Hz}\right)^{1 / 2}<0.01$, while under the high IC solution, $A_{*} \approx$ $0.07\left(\nu_{a} / 1.4 \mathrm{GHz}\right)^{-5 / 3}\left(\nu_{c} / 10^{15} \mathrm{~Hz}\right)^{(p / 2-2)}($ Sari \& Esin 2001).

\section{DISCUSSION AND CONCLUSIONS}

GRB 011121, a relatively nearby burst $(z=0.36)$, appears to have a firm identification of an underlying SN component (Paper I). Here we present early-time NIR and comprehensive dual-frequency centimeter-wave observations of the afterglow. Thanks to the NIR data, we have been able to accurately measure the considerable Galactic extinction toward the burst, $A_{V}=1.16 \pm 0.25 \mathrm{mag}$, which is significantly smaller than that derived from extrapolations of the IR maps (Schlegel et al. 1998). Our value of $A_{V}$ is an important parameter in the modeling of the underlying SN component (Paper I).

If indeed long-duration gamma-ray events such as GRB 011121 are linked to $\mathrm{SNe}$, then the progenitors of GRBs are massive stars. Such stars possess strong winds, and one expects to see the signature of the wind-fed circumburst medium on the afterglow emission (Chevalier \& Li 1999). The optical/NIR data alone rule out an isotropic explosion in a constant circumburst medium model. The radio data firmly rule out a model in which a jet is fully developed at $t<0.5$ days but allow for a wind-fed circumburst medium. Thus, we can infer that the progenitor of GRB 011121 was a massive star, which inference is completely independent of the discovery of the underlying SN (Paper I).

In the collapsar model (MacFadyen, Woosley, \& Heger 2001), one expects the progenitors of GRBs to be massive stars that have lost their hydrogen envelopes, i.e., Wolf-Rayet stars. For such stars, $v_{w} \sim 10^{3} v_{w 3} \mathrm{~km} \mathrm{~s}^{-1}$. For the progenitor of GRB 011121 , we derive $A_{*} \approx 0.02$ and $\dot{M} \sim 2 \times 10^{-7} v_{w 3}^{-1} M_{\odot} \mathrm{yr}^{-1}$. 
This $\dot{M}$ is consistent with that inferred for the progenitor of the Type Ic SN 1998bw $\left(2.5 \times 10^{-7} M_{\odot} \mathrm{yr}^{-1}\right.$; Li \& Chevalier 1999), which is thought to be associated with GRB 980425 based on the spatial and temporal coincidence (Galama et al. 1998) as well as its relativistic outflows (Kulkarni et al. 1998). However, this GRB, if associated with SN 1998bw, releases at least 3 orders of magnitude less energy in gamma rays compared with cosmological bursts (Galama et al. 1998) such as GRB 011121. So the relation of GRB 980425 to cosmological GRBs is unclear. Nevertheless, we make the following observation without further prejudice: the gamma-ray time history (Fig. 1) is of similar duration, smoothness, and shape (with a couple of spikes superposed) as that of GRB 980425. The massloss rate that we derive is about 2 orders of magnitude smaller than the value one expects for a $10 M_{\odot}$ Wolf-Rayet star (Wellstein \& Langer 1999), which may simply reflect the uncertainties in our analysis.

The current data clearly rule out a jet break on the timescale of the optical data, $t_{j} \gtrsim 2$ days, and the radio data require $t_{j} \gtrsim 7$ days. Using the formulation of Frail et al. (2001), the opening angle of the jet must be wider than $\theta_{j}>10^{\circ}$, and hence the true energy release is larger than $5 \times 10^{50} \mathrm{ergs}$. This lower limit is consistent with the clustering of energies around $5 \times$ $10^{50}$ ergs found by Frail et al. (2001).

Further improvements to the modeling are possible by including the BeppoSAX measurement of the X-ray afterglow (Piro et al. 2001); the X-ray flux will pin down $\nu_{c}$ quite well and may enable the high and low IC solutions to be distinguished, resulting in a more precise estimate of the progenitor mass-loss rate. We also note that the radio fluxes given in Table 2 suffer from strong variability (due to interstellar scintillation). Here we have used the mean fluxes, and in a later paper we intend to report detailed analysis of the scintillation and include the variability as a part of our afterglow modeling, in particular as a way to constrain the size of the afterglow region (see Frail et al. 1997).
GRB 011121, in which we see both evidence of a windstratified circumburst medium and an underlying SN (Paper I), is currently unique among all GRBs with afterglows studied to the present date. This prompts the question of why such evidences are not seen in all GRBs with afterglows. Leaving aside the immense observational effort needed to identify underlying $\mathrm{SNe}$, the absence of SN components in other GRBs can be explained by appealing to the well-known wide diversity in luminosity of Type Ib/c SNe (Mazzali et al. 2002).

The absence of clear wind signatures in some afterglows motivated Chevalier \& Li (2000) to suggest two different classes of progenitors. However, here again, we note an immense observational effort is needed to identify the signature of a wind. For GRB 011121, the abundance of our NIR data allowed us to accurately measure $\alpha$ and $\beta$ (in particular), in spite of the large foreground extinction. Combining these with our radio observations helped us to rule out a constant density model. Thus, GRB 011121 vividly demonstrates the importance of broadband optical/NIR observations and radio observations in inferring the circumburst medium.

P. A. P. gratefully acknowledges an Alex Rodgers Traveling Scholarship. GRB research at Caltech (S. R. K., S. G. D., F. A. H., R. S.) is supported by grants from NSF and NASA. J. S. B. is a Fannie and Hertz Foundation Fellow. R. S. holds a holds a Senior Fairchild Fellowship. K. H. is grateful for Ulysses support under JPL contract 958056 and for IPN support under NASA grants FDNAG 5-11451 and NAG 5-10710. We thank R. Chevalier for useful discussion and E. Mazets, $\mathrm{S}$. Golenetskii, and the Konus team for the use of the Konus data. Finally, we thank the staff of Las Campanas Observatory and the Australia Telescope National Facility for their assistance, and we applaud the heroic efforts of the staff of the AAT in obtaining these observations during the commissioning of IRIS2.

\section{REFERENCES}

Berger, E., et al. 2001, ApJ, 556, 556

Bloom, J. S., et al. 1999, Nature, 401, 453 2002, ApJ, 572, L45 (Paper I)

Cardelli, J. A., Clayton, G. C., \& Mathis, J. S. 1989, ApJ, 345, 245

Chevalier, R. A., \& Li, Z.-Y. 1999, ApJ, 520, L29

2000, ApJ, 536, 195

Fitzpatrick, E. L., \& Massa, D. 1988, ApJ, 328, 734

Frail, D. A., Kulkarni, S. R., Nicastro, S. R., Feroci, M., \& Taylor, G. B. 1997, Nature, 389, 261

Frail, D. A., et al. 2001, ApJ, 562, L55

2000, ApJ, 534, 559

Galama, T. J., et al. 2000, ApJ, 536, 185

1998, Nature, 395, 670

Garnavich, P. M., Holland, S. T., Jha, S., Kirshner, R. P., Bersier, D., \& Stanek, K. Z. 2002, GCN Circ. 1273 (http://gcn.gsfc.nasa.gov/gcn/gcn3/1273.gcn3) Hawarden, T. G., Leggett, S. K., Letawsky, M. B., Ballantyne, D. R., \& Casali, M. M. 2001, MNRAS, 325, 563

Hurley, K., Cline, T., Guidorzi, C., Montanari, E., Frontera, F., \& Feroci, M. 2001, GCN Circ. 1148 (http://gen.gsfc.nasa.gov/gcn/gen3/1148.gcn3)

Infante, L., Garnavich, P. M., Stanek, K. Z., \& Wyrzykowski, L. 2001, GCN

Circ. 1152 (http://gcn.gsfc.nasa.gov/gcn/gcn3/1152.gcn3)

Jaunsen, A. O., et al. 2001, ApJ, 546, 127

Kulkarni, S. R., et al. 1998, Nature, 395, 663

Li, Z.-Y., \& Chevalier, R. A. 1999, ApJ, 526, 716

MacFadyen, A. I., Woosley, S. E., \& Heger, A. 2001, ApJ, 550, 410
Mazzali, P. A., et al. 2002, ApJ, submitted (astro-ph/0204007)

Olsen, K., Brown, M., Schommer, R., \& Stubbs, C. 2001, GCN Circ. 1157 (http://gcn.gsfc.nasa.gov/gcn/gcn3/1157.gcn3)

Persson, S. E., Murphy, D. C., Krzeminski, W. Roth, M., \& Rieke, M. J. 1998, AJ, 116, 2475

Phillips, M., Krisciunas, K., Garnavich, P., Holland, S., Jha, S., Stanek, K. Z., \& McCarthy, P. 2001, GCN Circ. 1164 (http://gcn.gsfc.nasa.gov/gcn/gcn3/ 1164.gcn3)

Piro, L. 2001, GCN Circ. 1147 (http://gcn.gsfc.nasa.gov/gcn/gen3/1147.gcn3) Piro, L., et al. 2001, GCN Circ. 1172 (http://gcn.gsfc.nasa.gov/gen/gcn3/ 1172.gcn3)

Reichart, D. E. 1999, ApJ, 521, L111

. 2001, ApJ, 553, 235

Sari, R., \& Esin, A. A. 2001, ApJ, 548, 787

Sari, R., Piran, T., \& Halpern, J. P. 1999, ApJ, 519, L17

Sari, R., Piran, T., \& Narayan, R. 1998, ApJ, 497, L17

Schlegel, D. J., Finkbeiner, D. P., \& Davis, M. 1998, ApJ, 500, 525

Stanek, K. Z., \& Wyrzykowski, L. 2001, GCN Circ. 1160 (http://gcn.gsfc .nasa.gov/gcn/gcn3/1160.gcn3)

Stetson, P. B. 1987, PASP, 99, 191

Wellstein, S., \& Langer, N. 1999, A\&A, 350, 148

Woosley, S. E. 1993, ApJ, 405, 273

Wyrzykowski, L., Stanek, K. Z., \& Garnavich, P. M. 2001, GCN Circ. 1150

(http://gen.gsfc.nasa.gov/gen/gen3/1150.gen3) 